\title{
The properties of the solution for a class of switched systems with internally forced switching
}

\author{
Huanting Li ${ }^{1}$ and Xiankang Chen ${ }^{2^{*}}$ (D)
}

\author{
"Correspondence: \\ xiankangchen@yeah.net \\ ${ }^{2}$ Department of Mathematics and \\ Computer Science, Liupanshui \\ Normal University, Liupanshui, \\ China \\ Full list of author information is \\ available at the end of the article
}

\begin{abstract}
In this paper, the dynamic behavior of a class of switched systems with internally forced switching (IFS) is investigated. By introducing the definitions of continuous dependence and differentiability, the continuous dependence and differentiability of the solution relative to the control function are obtained. In the past studies, the optimal control problem given by IFS mainly focused on a special class of controlled systems (the piece affine system). Our results lay a good foundation for studying the more general internally forced switching problem.
\end{abstract}

MSC: $34 \mathrm{C} 25 ; 34 \mathrm{~A} 12 ; 34 \mathrm{D} 23$

Keywords: Switched systems; Internally forced switching; Continuous dependence; Differentiability

\section{Introduction}

Recently, the switched system has been an object of increasing interest because of its wide applicability in many fields, such as vehicle's engine management systems [36], electrical power systems [7, 32], robot systems [13, 16], chemical [11, 22], and so on. For the recent results on its stability and stabilization, one can refer to the survey [24] and the references therein. For the recent results on stability of switched systems, one can read the reports $[8,9,20,21,35,42]$. In addition to stability and stabilization issues, its optimal control problem also has attracted researchers from various fields in science and engineering. There are both theoretical and computational results in the open literature. The available theoretical results usually extend the classical maximum principle or the dynamic programming approach to the switched system (see [29, 30, 34]). Namely, studying the behavior of the switched system is most important in studying its optimal control problem, particularly the continuous dependence and differentiability of the solution relative to the control function of the switched system.

As we all know, the switched system is a special class of hybrid systems consisting of several subsystems and a switching law. In general, as a controlled system, a switched system

(c) The Author(s) 2021. This article is licensed under a Creative Commons Attribution 4.0 International License, which permits use, sharing, adaptation, distribution and reproduction in any medium or format, as long as you give appropriate credit to the original author(s) and the source, provide a link to the Creative Commons licence, and indicate if changes were made. The images or other third party material in this article are included in the article's Creative Commons licence, unless indicated otherwise in a credit line to the material. If material is not included in the article's Creative Commons licence and your intended use is not permitted by statutory regulation or exceeds the permitted use, you will need to obtain permission directly from the copyright holder. To view a copy of this licence, visit http://creativecommons.org/licenses/by/4.0/. 
can be expressed as

$$
\dot{x}(t)=f_{\omega(t, x(t))}(x(t), u(t)), \quad t>0,
$$

where $\omega$ is the switching law valuing from $\mathscr{P}\left(\mathscr{P}\right.$ is an index set), $u \in \mathbb{R}^{m}$ is the input, and $f_{i}: \mathbb{R}^{n} \times \mathbb{R}^{m} \rightarrow \mathbb{R}^{n}, i \in \mathscr{P}$, is a family of known functions. According to the switching law, the switched system can be classified into two classes: the switched system with internally forced switching (IFS) or externally forced switching (EFS). The switching law of IFS is based on the information of the state and the current mode. In general, it can be a function of $t, x$, and $p$. The autonomous lane change systems is a typical class of switched systems with internally forced switching. Each mode of the autonomous lane change system can be described by a differential equation. Transitions between modes are abrupt and triggered by system states and its constantly changing surroundings including velocity, throttle angle, engaged gear, obstacles, traffic signs, pedestrian, and so on. Note that the switching times are not pre-set but dependent on the time and states, which implies that its switching law is a function of $t$ and $x$. The switching law of EFS is an exogenous input to the system such as the traffic signal lamp system. Its switching times are not dependent on the vehicle driving states but pre-set. Namely, the exogenous input of IFS is only $u$ while the exogenous input of EFS is a pair $(\omega, u)$. Furthermore, the optimal control problem given by the switched system can be divided into two kinds: internally forced switching problem and externally forced switching problem according to the above taxonomy. The internally forced switching problem is finding an admissible control $u$ while the externally forced switching problem is finding an admissible control pair $(\omega, u)$. In addition, numerous examples indicate that IFS is more effective in modeling the smart system, such as self-driving cars [3,33], switched Hopfield neural networks [14, 41], memristive neural networks [26], Toxin systems, and so on.

The above analysis shows that IFS is relatively simple and smart. However, the optimal control problem of the switched system focuses on the externally forced switching problem and obtains great achievements (see [10,12, 17, 37-39]). The existing literature of the externally forced switching problem mainly covers a special class of IFS (the piecewise affine system) (see $[2,15,18,28,31])$. The more general internally forced switching problem is still an open problem. The difficulty in this problem is that the solution of IFS does not have continuous dependence relative to the control due to its switching law. Naturally, the differentiability of the solution with respect to the control does not hold either. To make the phenomenon clear, we will give a simple example. We first define several functions as follows:

$$
f_{1}(t)=2 t-u, \quad t \in[0,+\infty), \quad f_{2}(t)=-2 t, \quad t \in[0,+\infty)
$$

and

$$
y_{1}(t)=1, \quad t \in[0,+\infty), \quad y_{2}(t)=0, \quad t \in[0,+\infty)
$$

Consider the following switched system with internally forced switching:

$$
\dot{x}(t)=f_{\omega(x)}(t), \quad t \geq t_{0},
$$


where the switching law $\omega$ is a piecewise constant function from $\mathbb{R}^{+}$to $\mathscr{P}$ having the form

$$
\omega(x)= \begin{cases}1, & \text { if } t \in \bigcup_{x(a)=y_{1}}[a, b), \\ 2, & \text { if } t \in \bigcup_{x(a)=y_{2}}[a, b) .\end{cases}
$$

The time interval $[a, b)$ satisfies the following conditions: (i) set $a=\tilde{t}$ if there exists $\tilde{t} \in$ $\left[t_{0},+\infty\right)$ such that $x(\tilde{t})=y_{i}, i \in \mathscr{P}$, and (ii) set

$$
b=\inf \left\{t \mid t \in(a,+\infty), x(t)=y_{j}, j \in \mathscr{P}, i \neq j\right\}
$$

if there exists $\bar{t} \in(a,+\infty)$ such that $x(\bar{t})=y_{j}, j \in \mathscr{P}, i \neq j$. Otherwise, let $b=+\infty$.

Denote by $x(\cdot ; u)$ the solution of $(2)$ corresponding to the control function $u$. Let the initial state of $(2)$ be $(0,1)$, then we have the following result:

$$
x(t ; 2)= \begin{cases}(t-1)^{2}, & t \in[0,1), \\ -t^{2}+1, & t \in[1,+\infty),\end{cases}
$$

and

$$
x\left(t ; 2-\frac{1}{n}\right)=(t-1)^{2}+\frac{1}{n} t .
$$

Furthermore, we derive that

$$
\lim _{n \rightarrow \infty}\left\|x(t ; 2)-x\left(t ; 2-\frac{1}{n}\right)\right\|_{L^{1}((0,1), \mathbb{R})}=1
$$

which implies that the solution $x(\cdot ; 2)$ of $(2)$ is not continuously dependent on the control function in the $L^{1}$ space. Naturally, we cannot expect the differentiability of the solution with respect to the control function either. As is known, the continuous dependence and differentiability of the solution with respect to the control function of the controlled system are the key to studying its optimal control problem. However, to our knowledge, the study of IFS is focused on the stability $[1,4,5,19,25,27,40]$. Compared with a large number of results for stability of IFS, only a few results have appeared on the existence and continuity of its solution. In particular, as far as we know, to date, except for [25] and [6] studying the existence of the solution of a class of IFSs, there has been no published papers on the continuous dependence and differentiability of the solution for IFS. In conclusion, the continuous dependence and differentiability of the solution for IFS are interesting and challenging problems.

Motivated by the ideas, in this paper we study the continuous dependence and differentiability of the solution with respect to the control of a class of switched systems with internally forced switching. Suppose that $f_{i}: \mathbb{R}^{+} \rightarrow \mathbb{R}$ is a family of known functions, $a$ and the warning line $y_{i}$ both are known constants in $\mathbb{R}$, and the control function $u \in L_{\mathrm{loc}}^{p}\left(\mathbb{R}^{+}, \mathbb{R}\right)$ where $i \in \mathscr{P}, \mathscr{P}=\{1,2\}$. In this paper, we deal with the following switched system with internally forced switching:

$$
\dot{x}(t)=a x(t)+f_{\omega(x)}(t)+u(t), \quad t \geq 0,
$$


where the switching law $\omega: \mathbb{R} \rightarrow \mathscr{P}$ is a piecewise constant function defined by

$$
\omega(x)= \begin{cases}1, & \text { if } t \in \bigcup_{x(a)=y_{1}}[a, b), \\ 2, & \text { if } t \in \bigcup_{x(a)=y_{2}}[a, b) .\end{cases}
$$

and $[a, b)$ is the same as (2). Throughout this paper, let $\left(0, y_{1}\right)$ be the initial state of IFS (3).

Our goal in the paper is to study the continuous dependence and differentiability of the solution relative to the control function $u$ for IFS (3). The rest of the paper is organized as follows. In Sect. 2, introducing some definitions, modifying some classical definitions, the continuous dependence of the solution for IFS (3) is discussed. In Sect. 3, the differentiability of the solution for IFS (3) is given.

Notations Throughout this paper, we use the following notations. $\mathbb{R}^{n}$ denotes the $n$ dimensional Euclidean space and $|x|$ denotes the Euclidean norm of a vector $x$. Take $\mathbb{R}^{+}=[0,+\infty)$ and $O_{y_{i}}^{\theta^{2}}=\overline{O\left(y_{i}, \theta^{2}\right)}$, where $\overline{O\left(y_{i}, \theta^{2}\right)}$ is the closed set centered at $y_{i}$ and of radius $\theta^{2}$. For any $U \subset \mathbb{R}^{+}, L^{p}(U, \mathbb{R})$ denotes the class of Lebesgue measurable functions $f$ from $U$ to $\mathbb{R}$ with $\|f\|_{L^{p}(U, \mathbb{R})}<\infty$, where $\|f\|_{L^{p}(U, \mathbb{R})}=\left(\int_{U}|f(t)|^{p} d t\right)^{\frac{1}{p}}(1 \leq p<\infty)$. Also, let $C(U, \mathbb{R})$ be the class of continuous functions $g$ from $U$ to $\mathbb{R}$ and $\|g\|_{C(U, \mathbb{R})}=\max _{t \in U}|g(t)|$.

\section{Continuous dependence of solution on the control}

In this section, we consider the continuous dependence of the solution of IFS (3) with respect to the control. It is found from example (2) that the solution of IFS might not have the continuous dependence relative to the control even in the $L^{1}$ space. A major reason for this is that there is a different number of switches on bounded time intervals under a small perturbation of the control. That is to say, small perturbations of the control can have a great impact on the switching times of the systems. In order to overcome the difficulties and obtain the continuous dependence of the solution of IFS (3) relative to the control, some preliminaries will be involved. According to our previous study [23], we have the following result.

Lemma 2.1 Suppose that $u \in L_{\mathrm{loc}}^{p}\left(\mathbb{R}^{+}, \mathbb{R}\right)$ and $f_{i} \in L_{\mathrm{loc}}^{p}\left(\mathbb{R}^{+}, \mathbb{R}\right)(p>1, i \in \mathscr{P})$, then IFS (3) admits a unique solution.

Let $x(\cdot ; u)$ be the solution of IFS (3) corresponding to the initial conditions $\left(0, y_{1}\right)$ and the control function $u$, then the solution of IFS (3) has the following form:

$$
\begin{aligned}
x(t ; u)= & e^{a t} y_{1}+\int_{0}^{t} e^{a(t-\tau)} u(\tau) d \tau+\sum_{\substack{0 \leq t_{k-1}<t_{k}<t \\
x\left(t_{k-1} ; u\right)=y_{i}}} \int_{t_{k-1}}^{t_{k}} e^{a(t-\tau)} f_{i}(\tau) d \tau \\
& +\int_{x\left(t_{k} ; u\right)=y_{j}} e^{\left.a\left(t_{k}, t\right] \tau\right)} f_{j}(\tau) d \tau,
\end{aligned}
$$

where $i, j \in \mathscr{P}, i \neq j, k \in \mathbb{N}^{+}$.

Furthermore, we introduce the definitions of the approximate solution and continuous dependence. 
Definition 2.1 A function $x_{\theta}(\cdot ; u) \in C\left(\mathbb{R}^{+}, \mathbb{R}\right)$ is said to be an approximate solution of IFS (3) if, for any fixed sufficiently small $\theta>0, x_{\theta}(\cdot ; u)$ satisfies the following integral equation:

$$
\begin{aligned}
x_{\theta}(t ; u)= & e^{a t} y_{1}+\int_{0}^{t} e^{a(t-\tau)} u(\tau) d \tau+\sum_{\substack{0 \leq t_{k-1}<t_{k}<t \\
x\left(t_{k-1} ; u\right) \in O_{y_{i}}}} \int_{t_{k-1}}^{t_{k}} e^{a(t-\tau)} f_{i}(\tau) d \tau \\
& +\int_{\substack{x\left(t_{k} ; u\right) \in O_{y_{j}} \\
\left[t_{k}, t\right]}} e^{a(t-\tau)} f_{j}(\tau) d \tau,
\end{aligned}
$$

where $i, j \in \mathscr{P}, i \neq j, k \in \mathbb{N}^{+}$.

Note that the approximate solution $x_{\theta}(\cdot ; u)$ is the solution of IFS (3), as $\theta=0$. Meanwhile, Lemma 2.1 shows that, for any sufficient small $\theta>0$, (3) admits a unique approximate solution $x_{\theta}(\cdot ; u)$ having the form (6) under the conditions of Lemma 2.1, and IFS (3) at most has a finite number of switches on every bounded time interval (see the literature [23] for detail). Let

$$
u_{n}(\cdot) \in L_{\mathrm{loc}}^{p}\left(\mathbb{R}^{+}, \mathbb{R}\right) \quad \text { with }\left\|u_{n}-u\right\|_{L^{p}} \rightarrow 0
$$

and $x_{\theta}\left(\cdot ; u_{n}\right)$ be an approximate solution of IFS (3) corresponding to the control function $u_{n}(\cdot)$. It can be seen from Definition 2.1 that $x_{\theta}\left(\cdot, u_{n}\right)$ and $x(\cdot)$ have the same number of irregular points on $[0, T]$ for any fixed $T>0$.

Definition 2.2 The solution $x(\cdot ; u)$ of IFS (3) is said to have continuous dependence relative to the control function $u$ if

(1)

$$
\lim _{\substack{\theta \rightarrow 0 \\\left\|u_{n}-u\right\|_{L^{p}} \rightarrow 0}} x_{\theta}\left(t ; u_{n}\right)=x(t ; u), \quad \text { if } x(t) \neq y_{i} \text { for all } i \in \mathscr{P}
$$

and (2) given any $\epsilon>0$ there is a closed set $I_{\epsilon} \subseteq[0, T]$ and $\delta>0$ such that $m\left([0, T] \backslash I_{\epsilon}\right)<\epsilon$ and

$$
\left|x_{\theta}\left(t ; u_{n}\right)-x(t ; u)\right|<\epsilon, \quad t \in I_{\epsilon}
$$

provided $\theta+\left\|u_{n}-u\right\|_{L^{p}}<\delta$, where $m\left([0, T] \backslash I_{\epsilon}\right)$ is the Lebesgue measures of the set $[0, T] \backslash I_{\epsilon}$.

Furthermore, we have the following result.

Theorem 2.1 If $u \in L_{\mathrm{loc}}^{p}\left(\mathbb{R}^{+}, \mathbb{R}\right)$ and $f_{i} \in L_{\mathrm{loc}}^{p}\left(\mathbb{R}^{+}, \mathbb{R}\right)(p>1, i \in \mathscr{P})$, then the solution of IFS (3) has continuous dependence relative to the control function $u$ in the sense of Definition 2.2.

Proof Firstly, it can be seen from Lemma 2.1 that IFS (3) at most has a finite number of switches on every bounded time interval $[0, T]$ for any fixed $T>0$ (see the literature [23] for detail). Namely, there are only two possibilities: Case (1): IFS (3) does not switch on 
$[0, T]$ and Case (2): IFS (3) switches at least one time on $[0, T]$. We will prove the result from the two cases.

In Case (1), according to the continuous dependence of the solution on the parameter for an ordinary differential equation, we obtain that $x(\cdot)$ has continuous dependence on the initial value in the classical sense, i.e.,

$$
\lim _{\substack{\theta \rightarrow 0 \\\left\|u_{k}-u\right\|_{L} \rightarrow 0}}\left\|x_{\theta}\left(\cdot ; u_{n}\right)-x(\cdot ; u)\right\|_{C\left(\left[t_{0}, T\right], \mathbb{R}\right)}=0
$$

In Case (2), without loss of generally, assume IFS (3) just switches $k$ times on $[0, T]$. For convenience, let $t_{m} \in[0, T](m=1,2, \ldots, k)$ be the switching times of IFS (3). From the definition of its switching law (see (4), we know that there exist some $i \in \mathscr{P}$ such that $x\left(t_{m} ; u\right)=y_{i}$, in which $i=1$ if $m$ is even and $i=2$ if $m$ is odd. Combining Lemma 2.1 with Definition 2.1, we have that IFS (3) has a unique approximate solution $x_{\theta}\left(\cdot ; u_{n}\right)$ for any sufficient small $\theta>0$ under the conditions of Theorem 2.1. Also, Definition 2.1 suggests that $x_{\theta}\left(\cdot ; u_{n}\right)$ and $x(\cdot ; u)$ have the same number of switching times on $[0, T]$. Let $t_{m}\left(\theta ; f_{j}\right)$ $(m=1,2, \ldots, k, j \in \mathscr{P})$ be the switching times of $x_{\theta}\left(\cdot ; u_{n}\right)$. Then, given any $\epsilon>0$, IFS (3) does not switch on $\left[0, t_{1}-\frac{\epsilon}{4 k}\right]$. Hence, there exists $\delta_{1}>0$ such that

$$
\left|x_{\theta}\left(t ; u_{n}\right)-x(t ; u)\right|<\epsilon
$$

for each $\theta+\left\|u_{n}-u\right\|_{L^{p}}<\delta_{1}, t \in\left[t_{0}, t_{1}-\frac{\epsilon}{4 k}\right]$, which means that

$$
\lim _{\theta+\left\|u_{n}-u\right\|_{L^{p}} \rightarrow 0} t_{1}\left(\theta ; f_{1}\right)=t_{1}
$$

Analogously, consider the time interval $\left[t_{1}+\frac{\epsilon}{4 k}, t_{2}-\frac{\epsilon}{4 k}\right]$. Recalling equation (5) and Definition 2.1, we have that

$$
\begin{aligned}
\left|x_{\theta}\left(t ; u_{n}\right)-x(t ; u)\right| & \\
= & \mid e^{a\left(t-t_{1}\left(\theta ; f_{1}\right)\right)} \tilde{y}_{2}+\int_{t_{1}\left(\theta ; f_{1}\right)}^{t} e^{a(t-\tau)}\left(u_{n}(\tau)+f_{2}(\tau)\right) d \tau \\
& -\left[e^{a\left(t-t_{1}\right)} y_{2}+\int_{t_{1}}^{t} e^{a(t-\tau)}\left(u(\tau)+f_{2}(\tau)\right) d \tau\right] \mid \\
\leq & \left|e^{a\left(t-t_{1}\right)} y_{2}-e^{a\left(t-t_{1}\right)} \tilde{y}_{2}\right|+\left|e^{a\left(t-t_{1}\right)} \tilde{y}_{2}-e^{a\left(t-t_{1}\left(\theta ; f_{1}\right)\right)} \tilde{y}_{2}\right| \\
& +\int_{t_{1}\left(\theta ; f_{1}\right)}^{t} e^{a(t-\tau)}\left|u(\tau)-u_{n}(\tau)\right| d \tau \\
\leq & e^{a\left(t-t_{1}\right)}\left|y_{2}-\tilde{y}_{2}\right|+e^{a\left(t-t_{1}\right)}\left|a \tilde{y}_{2}\left(t_{1}-t_{1}\left(\theta ; f_{1}\right)\right)\right|+\int_{t_{1}}^{t_{1}\left(\theta ; f_{1}\right)} e^{a(t-\tau)}\left|u(\tau)+f_{2}(\tau)\right| d \tau \\
& +\int_{t_{1}\left(\theta ; f_{1}\right)}^{t} e^{a(t-\tau)}\left|u(\tau)-u_{n}(\tau)\right| d \tau \\
\leq & e^{a T}\left[\theta^{2}+\left|a \tilde{y}_{2}\left(t_{1}-t_{1}\left(\theta ; f_{1}\right)\right)\right|+\int_{t_{1}}^{t_{1}\left(\theta ; f_{1}\right)}\left|u(\tau)+f_{2}(\tau)\right| d \tau\right. \\
& \left.+\int_{t_{1}\left(\theta ; f_{1}\right)}^{t}\left|u(\tau)-u_{n}(\tau)\right| d \tau\right]
\end{aligned}
$$


for each $t \in\left[t_{1}+\frac{\epsilon}{4 k}, t_{2}-\frac{\epsilon}{4 k}\right]$. Using Hölder's inequality, we have that

$$
\left|x_{\theta}\left(t ; u_{n}\right)-x(t ; u)\right| \leq e^{a T}\left[\theta^{2}+\left(\left|a \tilde{y}_{2}\right|+\left\|u+f_{2}\right\|_{L^{p}}\right)\left|t_{1}-t_{1}\left(\theta ; f_{1}\right)\right|+T^{\frac{1}{p}}\left\|u-u_{n}\right\|_{L^{p}}\right]
$$

for all $t \in\left[t_{1}+\frac{\epsilon}{4 k}, t_{2}-\frac{\epsilon}{4 k}\right]$. Furthermore, it can be seen from (7) and (8) that there exists $\delta_{2}>0\left(\delta_{2}<\delta_{1}\right)$ such that

$$
\left|x_{\theta}\left(t ; u_{n}\right)-x(t ; u)\right|<\epsilon, \quad \text { for all } t \in\left[t_{1}+\frac{\epsilon}{4 k}, t_{2}-\frac{\epsilon}{4 k}\right] \text { and } \theta+\left\|u-u_{n}\right\|_{L^{p}}<\delta_{2}
$$

and

$$
\lim _{\theta+\left\|u-u_{n}\right\|_{L} p \rightarrow 0} t_{2}\left(\theta ; f_{1}\right)=t_{2}
$$

In general, we consider the time interval $\left[t_{m}+\frac{\epsilon}{4 k}, t_{m+1}-\frac{\epsilon}{4 k}\right](m=1,2, \ldots, k-1)$ or $\left[t_{k}+\right.$ $\left.\frac{\epsilon}{4 k}, T\right]$. Combined (5) with Definition 2.1, we obtain that

$$
\begin{aligned}
\left|x_{\theta}\left(t ; u_{n}\right)-x(t ; u)\right| & \mid e^{a\left(t-t_{m}\left(\theta ; f_{j}\right)\right)} \tilde{y}_{i}+\int_{t_{m}\left(\theta ; f_{j}\right)}^{t} e^{a(t-\tau)}\left(u_{n}(\tau)+f_{i}(\tau)\right) d \tau \\
& -\left[e^{a\left(t-t_{m}\right)} y_{i}+\int_{t_{m}}^{t} e^{a(t-\tau)}\left(u(\tau)+f_{i}(\tau)\right) d \tau\right] \mid \\
\leq & \left|e^{a\left(t-t_{m}\right)} y_{i}-e^{a\left(t-t_{m}\right)} \tilde{y}_{i}\right|+\left|e^{a\left(t-t_{m}\right)} \tilde{y}_{i}-e^{a\left(t-t_{m}\left(\theta ; f_{j}\right)\right)} \tilde{y}_{i}\right| \\
& +\int_{t_{m}\left(\theta ; f_{j}\right)}^{t} e^{a(t-\tau)}\left|u(\tau)-u_{n}(\tau)\right| d \tau \\
\leq & e^{a\left(t-t_{m}\right)}\left|y_{i}-\tilde{y}_{i}\right|+e^{a\left(t-t_{m}\right)}\left|a \tilde{y}_{i}\left(t_{m}-t_{m}\left(\theta ; f_{j}\right)\right)\right|+\int_{t_{m}}^{t_{m}\left(\theta ; f_{j}\right)} e^{a(t-\tau)}\left|u(\tau)+f_{i}(\tau)\right| d \tau \\
& +\int_{t_{m}\left(\theta ; f_{j}\right)}^{t} e^{a(t-\tau)}\left|u(\tau)-u_{n}(\tau)\right| d \tau \\
\leq & e^{a T\left[\theta^{2}+\left|a \tilde{y}_{i}\left(t_{m}-t_{m}\left(\theta ; f_{j}\right)\right)\right|+\int_{t_{m}}^{t_{m}\left(\theta ; f_{j}\right)}\left|u(\tau)+f_{i}(\tau)\right| d \tau\right.} \\
& \left.+\int_{t_{m}\left(\theta ; f_{j}\right)}^{t}\left|u(\tau)-u_{n}(\tau)\right| d \tau\right]
\end{aligned}
$$

for each $t \in\left[t_{m}+\frac{\epsilon}{4 k}, t_{m+1}-\frac{\epsilon}{4 k}\right] \cup\left[t_{k}+\frac{\epsilon}{4 k}, T\right]$, where $i=1$ if $m$ is even and $i=2$ if $m$ is odd. Using Hölder's inequality, we have that

$$
\left|x_{\theta}\left(t ; u_{n}\right)-x(t ; u)\right| \leq e^{a T}\left[\theta^{2}+\left(\left|a \tilde{y}_{i}\right|+\left\|u+f_{i}\right\|_{L^{p}}\right)\left|t_{m}-t_{m}\left(\theta ; f_{j}\right)\right|+T^{\frac{1}{p}}\left\|u-u_{n}\right\|_{L^{p}}\right]
$$

for all $t \in\left[t_{m}+\frac{\epsilon}{4 k}, t_{m+1}-\frac{\epsilon}{4 k}\right] \cup\left[t_{k}+\frac{\epsilon}{4 k}, T\right]$. Furthermore, there exists a constant $\delta_{m+1}>0$ $\left(\delta_{m+1}<\delta_{m}\right)$ such that

$$
\left|x_{\theta}\left(t ; u_{n}\right)-x(t ; u)\right|<\epsilon \quad \text { for all } t \in\left[t_{m}+\frac{\epsilon}{4 k}, t_{m+1}-\frac{\epsilon}{4 k}\right] \text { and } \theta+\left\|u-u_{n}\right\|_{L^{p}}<\delta_{m+1}
$$


and

$$
\lim _{\theta+\left\|u-u_{n}\right\|_{L^{p}} \rightarrow 0} t_{m+1}\left(\theta ; f_{i}\right)=t_{m+1} .
$$

Now define

$$
I_{\epsilon}=\left[t_{0}, t_{1}-\frac{\epsilon}{4 k}\right] \cup\left(\bigcup_{m=1}^{k-1}\left[t_{m}+\frac{\epsilon}{4 k}, t_{m+1}-\frac{\epsilon}{4 k}\right]\right) \cup\left[t_{k}+\frac{\epsilon}{4 k}, T\right] .
$$

It is easy to see $m\left(\left[t_{0}, T\right] \backslash I_{\epsilon}\right)=\frac{\epsilon}{2}<\epsilon$, and there is $\delta=\delta_{k}>0$ such that the inequality

$$
\left|x_{\theta}\left(t ; u_{n}\right)-x(t ; u)\right|<\epsilon
$$

holds for each $t \in I_{\epsilon}$ and $\theta+\left\|u-u_{n}\right\|_{L^{p}} \in[0, \delta)$. This completes the proof of Theorem 2.1.

\section{Differentiability of solution with respect to the control}

In this section, we discuss the differentiability of the solution relative to the control function for IFS (3). Before that, we make some preparations. For convenience, we consider the smooth optimal control problem, namely the allowable control set $\mathscr{U}([0, T] ; \mathbb{R}) \subset$ $C([0, T] ; \mathbb{R})$. Suppose that $(\bar{x}, \bar{u})$ is the solution of the smooth optimal control problem given by IFS (3), where the optimal trajectory $\bar{x}(\cdot ; \bar{u}(\cdot))$ is the solution of IFS (3) corresponding to the optimal control $\bar{u}$. For any $\alpha \in \mathbb{R}$ and $u(\cdot) \in \mathscr{U}([0, T] ; \mathbb{R})$, we have that $u^{\alpha}(\cdot)=\bar{u}(\cdot)+\alpha u(\cdot) \in \mathscr{U}([0, T] ; \mathbb{R})$. Furthermore, it can be seen from Lemma 2.1 that IFS (3) has a unique approximate solution $u_{\alpha}\left(\cdot ; u^{\alpha}\right)$. From IFS (2), we can claim that IFS may be not continuous dependence relative to the control function. Naturally, we cannot expect to the differentiability of the solution relative to the control function. Hence, we need to modify the classical definition of the variation.

Definition 3.1 The solution $x(\cdot ; u)$ of IFS (3) is said to be Gâteaux differentiable relative to $u(\cdot)$ in the direction of the function $u^{\alpha}(\cdot)$ if

$$
\lim _{\alpha \searrow 0} \frac{x_{\alpha}\left(t ; u^{\alpha}\right)-x(t ; u)}{\alpha}
$$

exists for all $t$ such that $x(t ; u) \neq y_{i}$ for all $i \in \mathscr{P}$. Furthermore, let

$$
\varphi\left(t ; u^{\alpha}\right)= \begin{cases}\lim _{\alpha \searrow 0} \frac{x_{\alpha}\left(t ; u^{\alpha}\right)-x(t ; u)}{\alpha}, & x(t ; u) \neq y_{i} \text { for all } i \in \mathscr{P}, \\ \lim _{s \nearrow t} \lim _{\alpha \searrow 0} \frac{x_{\alpha}\left(t ; u^{\alpha}\right)-x(t ; u)}{\alpha}, & x(t)=y_{i} \text { for some } i \in \mathscr{P},\end{cases}
$$

then $\varphi\left(\cdot ; u^{\alpha}\right)$ is called the Gâteaux derivative of the solution $x(\cdot ; u)$ relative to the control function $u(\cdot)$ in the direction of $u^{\alpha}(\cdot)$.

To obtain the differentiability of the solution relative to the control function for IFS (3), we need the following lemma. For this purpose, we first define several functions as follows:

$$
\tilde{y}(\alpha)= \begin{cases}y_{i}-\alpha^{2}, & \text { if } y_{i}>y_{j}, \\ y_{i}+\alpha^{2}, & \text { if } y_{i}<y_{j}\end{cases}
$$


where $i, j \in \mathscr{P}$,

$$
H(\alpha, t)=x_{\alpha}\left(t ; u^{\alpha}\right)-\tilde{y}(\alpha)
$$

$h_{t}(\alpha)$ denotes the solution of $H(\theta, t)=0$.

Furthermore, we get the following lemma.

Lemma 3.1 If $f_{i} \in([0,+\infty), \mathbb{R})$ and $f_{i}(t)+\bar{u}(t) \neq a y_{j}$ for all $t \in[0,+\infty)$ and $i, j \in \mathscr{P}$, then there is $\delta>0$ such that $h_{t}$ is differentiable on $[0, \delta]$ and its derivative is given by

$$
\dot{h}_{t_{m}}(0)= \begin{cases}-\frac{\int_{0}^{t_{1}} e^{a\left(t_{1}-\tau\right)} u(\tau) d \tau}{f_{1}\left(t_{1}\right)+a y_{2}+\bar{u}\left(t_{1}\right)}, & m=1, \\ -\frac{\int_{0}^{t_{m}} e^{a\left(t_{m}-\tau\right)} u(\tau) d \tau+\sum_{i=1}^{m-1}(-1)^{i+1} \dot{h}_{t_{i}}(0) e^{a\left(t_{m}-t_{i}\right)}\left(f_{1}\left(t_{i}\right)-f_{2}\left(t_{i}\right)\right)-\dot{\tilde{y}}(\alpha)}{f_{i}\left(t_{m}\right)+a y_{j}+\bar{u}\left(t_{m}\right)}, & m=2,3, \ldots, k,\end{cases}
$$

where $i=2, j=1$ if $m$ is even and $i=1, j=2$ if $m$ is odd.

Proof We can deduce from Theorem 2.1 that there exists $\delta>0$ such that $h_{t}:[0, \delta] \rightarrow O(t)$ is a function for all $\alpha \in[0, \delta]$ and $h_{t}(0)=t$, if $x(t)=y_{i}$ for some $i \in \mathscr{P}$, where $O(t)$ denotes some neighborhood of $t$. Furthermore, we have that

$$
x_{\alpha}\left(h_{t_{m}}(\alpha) ; u^{\alpha}\right)=\tilde{y}(\alpha) \quad \text { for all } \alpha \in[0, \delta], m=1,2, \ldots, k,
$$

which implies

$$
H\left(\alpha, h_{t_{m}}(\alpha)\right)=0 \quad \text { for all } \alpha \in[0, \delta], m=1,2, \ldots, k \text {. }
$$

Since

$$
\frac{\partial H(\alpha, t)}{\partial t}=\frac{\partial\left[x_{\alpha}\left(t ; u^{\alpha}\right)-\tilde{y}(\alpha)\right]}{\partial t}=a x_{\alpha}\left(t ; u^{\alpha}\right)+u^{\alpha}(t)+f_{i}(t) \quad \text { for all } \alpha \in[0, \delta],
$$

then it can be seen from $f_{i}(\cdot) \in C([0, T] ; \mathbb{R})$ and $u^{\alpha}(\cdot) \in C([0, T] ; \mathbb{R})$ that $\frac{\partial H(\alpha, t)}{\partial t}$ is continuous on $[0, \delta] \times\left[h_{t_{m}}, h_{t_{m+1}}\right]$. Combined with $f_{i}(t)+\bar{u}(t) \neq-a y_{i}$ for all $i \in \mathscr{P}$, we have that

$$
\begin{aligned}
\left.\frac{\partial H(\alpha, t)}{\partial t}\right|_{t=h_{t_{m}}(\alpha)} & =\left.\frac{\partial\left[x_{\alpha}\left(t ; u^{\alpha}\right)-\tilde{y}(\alpha)\right]}{\partial t}\right|_{t=h_{t_{m}}(\alpha)} \\
& =f_{i}\left(h_{t_{m}}(\alpha)\right)+a \tilde{y}(\alpha)+u^{\alpha}\left(h_{t_{1}}(\alpha)\right) \neq 0,
\end{aligned}
$$

where $m=1,2, \ldots, k, i \in \mathscr{P}$. Otherwise, for the time interval $\left[0, h_{t_{1}}(\alpha)\right)$, we obtain that

$$
\begin{aligned}
& H_{\alpha}(\alpha, t) \\
& =\lim _{\xi \rightarrow 0} \frac{H(\alpha+\xi, t)-H(\alpha, t)}{\xi} \\
& =\lim _{\xi \rightarrow 0} \frac{x_{\alpha+\xi}\left(t ; u^{\alpha+\xi}\right)-x_{\alpha}\left(t ; u^{\alpha}\right)}{\xi}-\dot{\tilde{y}}(\alpha) \\
& =\lim _{\xi \rightarrow 0} \frac{e^{a t} y_{1}+\int_{0}^{t} e^{a(t-\tau)}\left(u^{\alpha+\xi}(\tau)+f_{1}(\tau)\right) d \tau-\left[e^{a t} y_{1}+\int_{0}^{t} e^{a(t-\tau)}\left(u^{\alpha}(\tau)+f_{1}(\tau)\right) d \tau\right]}{\xi}
\end{aligned}
$$




$$
\begin{aligned}
& -\dot{\tilde{y}}(\alpha) \\
= & \int_{0}^{t} e^{a(t-\tau)} u(\tau) d \tau-\dot{\tilde{y}}(\alpha) .
\end{aligned}
$$

The above implies that the continuous partial derivative of $H(\alpha, t)$ with respect to $\alpha$ exists on $[0, \delta] \times\left[0, h_{t_{1}}(\alpha)\right)$. Furthermore, we induce from (11) and (12) that

$$
\dot{h}_{t_{1}}(\alpha)=-\left.\frac{\frac{\partial H(\alpha, t)}{\partial \alpha}}{\frac{\partial H(\alpha, t)}{\partial t}}\right|_{t=h_{t_{1}}(\alpha)}=-\frac{\int_{0}^{h_{t_{1}}(\alpha)} e^{a\left(h_{t_{1}}(\alpha)-\tau\right)} u(\tau) d \tau-\dot{\tilde{y}}(\alpha)}{f_{1}\left(h_{t_{1}}(\alpha)\right)+a \tilde{y}(\alpha)+u^{\alpha}\left(h_{t_{1}}(\alpha)\right)}
$$

and

$$
\dot{h}_{t_{1}}(0)=-\frac{\int_{0}^{t_{1}} e^{a\left(t_{1}-\tau\right)} u(\tau) d \tau}{f_{1}\left(t_{1}\right)+a y_{2}+\bar{u}\left(t_{1}\right)} .
$$

Secondly, when $t \in\left(h_{t_{1}}(\alpha), h_{t_{2}}(\alpha)\right)$, we can reduce that

$$
\begin{aligned}
& H_{\alpha}(\alpha, t) \\
& =\frac{\partial}{\partial \alpha}\left(e^{a t} y_{1}+\int_{0}^{t} e^{a(t-\tau)} u^{\alpha}(\tau) d \tau+\int_{0}^{h_{t_{1}(\alpha)}} e^{a(t-\tau)} f_{1}(\tau) d \tau+\int_{h_{t_{1}}(\alpha)}^{t} e^{a(t-\tau)} f_{2}(\tau) d \tau\right) \\
& \quad-\dot{\tilde{y}}(\alpha) \\
& =\int_{0}^{t} e^{a(t-\tau)} u(\tau) d \tau+\dot{h}_{t_{1}}(\alpha) e^{a\left(t-h_{t_{1}}(\alpha)\right)}\left[f_{1}\left(h_{t_{1}}(\alpha)\right)-f_{2}\left(h_{t_{1}}(\alpha)\right)-\dot{\tilde{y}}(\alpha)\right.
\end{aligned}
$$

which implies that the continuous partial derivative of $H(\alpha, t)$ with respect to $\alpha$ exists on $[0, \delta] \times\left[h_{t_{1}}(\alpha), h_{t_{2}}(\alpha)\right)$. Furthermore, we induce from (11) and (13) that

$$
\dot{h}_{t_{2}}(\alpha)=-\frac{\int_{0}^{h_{t_{2}}(\alpha)} e^{a\left(h_{t_{2}}(\alpha)-\tau\right)} u(\tau) d \tau+\dot{h}_{t_{1}}(\alpha) e^{a\left(h_{t_{2}}(\alpha)-h_{t_{1}}(\alpha)\right)}\left(f_{1}\left(h_{t_{1}}(\alpha)\right)-f_{2}\left(h_{t_{1}}(\alpha)\right)\right)-\dot{\tilde{y}}(\alpha)}{f_{2}\left(h_{t_{2}}(\alpha)\right)+a \tilde{y}(\alpha)+u^{\alpha}\left(h_{t_{2}}(\alpha)\right)}
$$

and

$$
\dot{h}_{t_{2}}(0)=-\frac{\int_{0}^{t_{2}} e^{a\left(t_{2}-\tau\right)} u(\tau) d \tau+\dot{h}_{t_{1}}(0) e^{a\left(t_{2}-t_{1}\right)}\left(f_{1}\left(t_{1}\right)-f_{2}\left(t_{1}\right)\right)}{f_{2}\left(h_{t_{2}}(\alpha)\right)+a y_{1}+\bar{u}\left(t_{2}\right)} .
$$

In general, for any $t \in\left(h_{t_{m}}(\alpha), h_{t_{m+1}}(\alpha)\right)(m=1,2, \ldots, k-1)$ or $t \in\left(h_{t_{k}}(\alpha), T\right]$, we have

$$
\begin{aligned}
& H_{\alpha}(\alpha, t) \\
& =\frac{\partial}{\partial \alpha}\left(e^{a t} y_{1}+\int_{0}^{t} e^{a(t-\tau)} u^{\alpha}(\tau) d \tau+\sum_{i=1}^{m} \int_{h_{t_{i-1}}(\alpha)}^{h_{t_{i}}(\alpha)} e^{a(t-\tau)} f_{p}(\tau) d \tau\right. \\
& \left.\quad+\int_{h_{t_{m}}(\alpha)}^{t} e^{a(t-\tau)} f_{j}(\tau) d \tau\right) \\
& \quad-\quad \dot{\tilde{y}}(\alpha) \\
& =\int_{0}^{t} e^{a(t-\tau)} u(\tau) d \tau+\sum_{i=1}^{m}(-1)^{i+1} \dot{h}_{t_{i}}(\alpha) e^{a\left(t-h_{t_{i}}(\alpha)\right)}\left(f_{1}\left(h_{t_{i}}(\alpha)\right)-f_{2}\left(h_{t_{i}}(\alpha)\right)-\dot{\tilde{y}}(\alpha),\right.
\end{aligned}
$$


where $p=1$ if $i$ is odd and $p=2$ if $i$ is even $j \in \mathscr{P}$ and $j \neq p$, which implies that the continuous partial derivative of $H(\epsilon, t)$ with respect to $\epsilon$ exists on $[0, \delta] \times\left(h_{t_{m}}(\epsilon), h_{t_{m+1}}(\epsilon)\right)$. Furthermore, from the implicit function theory, (11), and (14), we discover that

$$
\begin{aligned}
& \dot{h}_{t_{m+1}}(\alpha) \\
& =-\frac{\int_{0}^{h_{t_{m+1}}(\alpha)} e^{a\left(h_{t_{m+1}}(\alpha)-\tau\right)} u(\tau) d \tau+\sum_{i=1}^{m}(-1)^{i+1} \dot{h}_{t_{t}}(\alpha) e^{a\left(h_{t_{m+1}}(\alpha)-h_{t_{i}}(\alpha)\right)}\left(f_{1}\left(h_{t_{i}}(\alpha)\right)-f_{2}\left(h_{t_{i}}(\alpha)\right)\right)-\dot{\tilde{y}}(\alpha)}{f_{i}\left(h_{t_{m+1}}(\alpha)\right)+a \tilde{y}(\alpha)+u^{\alpha}\left(h_{t_{m+1}}(\alpha)\right)}
\end{aligned}
$$

and

$$
\dot{h}_{t_{m+1}}(0)=-\frac{\int_{0}^{t_{m+1}} e^{a\left(t_{m+1}-\tau\right)} u(\tau) d \tau+\sum_{i=1}^{m}(-1)^{i+1} \dot{h}_{t_{i}}(0) e^{a\left(t_{m+1}-t_{i}\right)}\left(f_{1}\left(t_{i}\right)-f_{2}\left(t_{i}\right)\right)-\dot{\tilde{y}}(\alpha)}{f_{i}\left(t_{m+1}\right)+a y_{j}+\bar{u}\left(t_{m+1}\right)}
$$

where $i=1, j=2$ if $m$ is even and $i=2, j=1$ if $m$ is odd. This completes the proof of Lemma 3.1.

Based on Lemma 3.1 and Definition 3.1, we will give our final result.

Theorem 3.1 Suppose that $f_{i} \in([0,+\infty), \mathbb{R})$ and $f_{i}(t)+\bar{u}(t) \neq$ ay $y_{j}$ for all $t \in[0,+\infty)$ and $i, j \in \mathscr{P}$, then for any $u(\cdot) \in \mathscr{U}([0, T] ; \mathbb{R})$, the solution $\bar{x}(\cdot ; \bar{u})$ of IFS (3) is Gâteaux differentiable at $\bar{u}(\cdot)$ in the direction of $u(\cdot)$ in the sense of Definition 3.1. Moreover, its derivative $\varphi$ satisfies the following impulsive differential equation:

$$
\begin{cases}\dot{\varphi}\left(t ; u^{\alpha}\right)=a \varphi\left(t ; u^{\alpha}\right)+u(t), & t \in\left(t_{0}, T\right], x(t) \neq y_{p}(p \in \mathscr{P}) . \\ \varphi\left(t+; u^{\alpha}\right)=\varphi\left(t ; u^{\alpha}\right)+\dot{h}_{t}(0)\left[f_{j}(t)-f_{i}(t)\right], & x(t)=y_{i}(i, j \in \mathscr{P}, i \neq j), \\ \varphi\left(0 ; u^{\alpha}\right)=0 . & \end{cases}
$$

Proof For the number of the irregular points on $\left[t_{0}, T\right]$, there are only two possibilities which are Case $(1): \bar{x}(\cdot ; \bar{u})$ has no irregular point on $\left[t_{0}, T\right]$ and Case $(2): \bar{x}(\cdot ; \bar{u})$ has at least one irregular point on $\left[t_{0}, T\right]$. We will prove Theorem 3.1 from the two aspects.

Case (1), one can directly check that $x(\cdot)$ is Gâteaux differentiable and its Gâteaux derivative $\varphi$ (see Definition 3.1) is a solution of the following differential equation:

$$
\left\{\begin{array}{l}
\dot{\varphi}\left(t ; u^{\alpha}\right)=a \varphi\left(t ; u^{\alpha}\right)+u(t), \quad t \in(0, T] \\
\varphi\left(0 ; u^{\alpha}\right)=0
\end{array}\right.
$$

Case (2), from Definition 3.1, we first have that

$$
\begin{aligned}
\varphi\left(t ; u^{\alpha}\right) & =\lim _{\alpha \rightarrow 0} \frac{x_{\alpha}\left(t ; u^{\alpha}\right)-\bar{x}(t ; \bar{u})}{\alpha} \\
& =\lim _{\alpha \rightarrow 0} \frac{e^{a t} y_{1}+\int_{0}^{t} e^{a(t-\tau)}\left(f_{1}(\tau)+u^{\alpha}(\tau)\right) d \tau-\left[e^{a t} y_{1}+\int_{0}^{t} e^{a(t-\tau)}\left(f_{1}(\tau)+\bar{u}(\tau)\right) d \tau\right]}{\alpha} \\
& =\int_{0}^{t} e^{a(t-\tau)} u(\tau) d \tau, \quad \forall t \in\left(0, t_{1}\right) .
\end{aligned}
$$


Namely, $\varphi(\cdot)$ satisfies the following equation:

$$
\left\{\begin{array}{l}
\dot{\varphi}\left(t ; u^{\alpha}\right)=a \varphi\left(t ; u^{\alpha}\right)+u(t), \quad t \in\left(0, t_{1}\right) \\
\varphi\left(0 ; u^{\alpha}\right)=0
\end{array}\right.
$$

Meanwhile, when $h_{t_{1}}(\alpha)>t_{1}$, combining equation (3) with (9), we discover that

$$
\begin{aligned}
\lim _{\alpha \rightarrow 0} & \frac{x_{\alpha}\left(h_{t_{1}}(\alpha) ; u^{\alpha}\right)-\bar{x}\left(t_{1} ; \bar{u}\right)}{\alpha} \\
= & \lim _{\alpha \rightarrow 0} \frac{x_{\alpha}\left(h_{t_{1}}(\alpha) ; u^{\alpha}\right)-x_{\alpha}\left(t_{1} ; u^{\alpha}\right)}{\alpha}+\lim _{\alpha \rightarrow 0} \frac{x_{\alpha}\left(t_{1} ; u^{\alpha}\right)-\bar{x}\left(t_{1} ; \bar{u}\right)}{\alpha} \\
= & \lim _{\alpha \rightarrow 0} \frac{x_{\alpha}\left(h_{t_{1}}(\alpha) ; u^{\alpha}\right)-x_{\alpha}\left(t_{1} ; u^{\alpha}\right)}{h_{t_{1}}(\alpha)-t_{1}} \frac{h_{t_{1}}(\alpha)-t_{1}}{\alpha}+\varphi\left(t_{1} ; u^{\alpha}\right) \\
= & \dot{h}_{t_{1}}(0)\left(a y_{2}+f_{1}\left(t_{1}\right)+\bar{u}\left(t_{1}\right)\right)+\varphi\left(t_{1} ; u^{\alpha}\right) .
\end{aligned}
$$

Furthermore, we can reduce that

$$
\begin{aligned}
\varphi\left(t_{1}+; u^{\alpha}\right) & =\lim _{\alpha \rightarrow 0} \frac{x_{\alpha}\left(h_{t_{1}}(\alpha) ; u^{\alpha}\right)-\bar{x}\left(h_{t_{1}}(\alpha) ; \bar{u}\right)}{\alpha} \\
& =\lim _{\alpha \rightarrow 0} \frac{x_{\alpha}\left(h_{t_{1}}(\alpha) ; u^{\alpha}\right)-\bar{x}\left(t_{1} ; \bar{u}\right)}{\alpha}+\lim _{\alpha \rightarrow 0} \frac{\bar{x}\left(t_{1} ; \bar{u}\right)-\bar{x}\left(h_{t_{1}}(\alpha) ; \bar{u}\right)}{\alpha} \\
& =\dot{h}_{t_{1}}(0)\left(f_{1}\left(t_{1}\right)-f_{2}\left(t_{1}\right)\right)+\varphi\left(t_{1} ; u^{\alpha}\right) .
\end{aligned}
$$

Similarly, when $h_{t_{1}}(\alpha)<t_{1}$, we have that

$$
\begin{aligned}
\lim _{\alpha \rightarrow 0} \frac{x_{\alpha}\left(h_{t_{1}}(\alpha) ; u^{\alpha}\right)-\bar{x}\left(t_{1} ; \bar{u}\right)}{\alpha} \\
\quad=\lim _{\alpha \rightarrow 0} \frac{x_{\alpha}\left(h_{t_{1}}(\alpha) ; u^{\alpha}\right)-\bar{x}\left(h_{t_{1}}(\alpha) ; \bar{u}\right)}{\alpha}+\lim _{\alpha \rightarrow 0} \frac{\bar{x}\left(h_{t_{1}}(\alpha) ; \bar{u}\right)-\bar{x}\left(t_{1} ; \bar{u}\right)}{\alpha} \\
\quad=\varphi\left(t_{1} ; u^{\alpha}\right)+\lim _{\alpha \rightarrow 0} \frac{\bar{x}\left(h_{t_{1}}(\alpha) ; \bar{u}\right)-\bar{x}\left(t_{1} ; \bar{u}\right)}{h_{t_{1}}(\alpha)-t_{1}} \frac{h_{t_{1}}(\alpha)-t_{1}}{\alpha} \\
\quad=\varphi\left(t_{1} ; u^{\alpha}\right)+\dot{h}_{t_{1}}(0)\left(a y_{2}+f_{1}\left(t_{1}\right)+\bar{u}\left(t_{1}\right)\right),
\end{aligned}
$$

and

$$
\begin{aligned}
\varphi\left(t_{1}+; u^{\alpha}\right) & =\lim _{\alpha \rightarrow 0} \frac{x_{\alpha}\left(t_{1} ; u^{\alpha}\right)-\bar{x}\left(t_{1} ; \bar{u}\right)}{\alpha} \\
& =\lim _{\alpha \rightarrow 0} \frac{x_{\alpha}\left(t_{1} ; u^{\alpha}\right)-x_{\alpha}\left(h_{t_{1}}(\alpha) ; u^{\alpha}\right)}{\alpha}+\lim _{\alpha \rightarrow 0} \frac{x_{\alpha}\left(h_{t_{1}}(\alpha) ; u^{\alpha}\right)-\bar{x}\left(t_{1} ; \bar{u}\right)}{\alpha} \\
& =\dot{h}_{t_{1}}(0)\left(f_{1}\left(t_{1}\right)-f_{2}\left(t_{1}\right)\right)+\varphi\left(t_{1} ; u^{\alpha}\right) .
\end{aligned}
$$

In conclusion, we can claim that

$$
\lim _{\alpha \rightarrow 0} \frac{x_{\alpha}\left(h_{t_{1}}(\alpha) ; u^{\alpha}\right)-\bar{x}\left(t_{1} ; \bar{u}\right)}{\alpha}=\varphi\left(t_{1}\right)+\dot{h}_{t_{1}}(0)\left(a y_{2}+f_{1}\left(t_{1}\right)+\bar{u}\left(t_{1}\right)\right)
$$

and

$$
\varphi\left(t_{1}+; u^{\alpha}\right)=\dot{h}_{t_{1}}(0)\left(f_{1}\left(t_{1}\right)-f_{2}\left(t_{1}\right)\right)+\varphi\left(t_{1} ; u^{\alpha}\right) .
$$


Analogously, we consider $\left(t_{1}, t_{2}\right)$. From the following equation

$$
\begin{aligned}
& \varphi\left(t ; u^{\alpha}\right) \\
& =\lim _{\alpha \rightarrow 0} \frac{x\left(t ; u^{\alpha}\right)-\bar{x}(t ; \bar{u})}{\alpha} \\
& =\lim _{\alpha \rightarrow 0} \frac{e^{a\left(t-h_{t_{1}}(\alpha)\right)} \tilde{y}(\alpha)+\int_{h_{t_{1}}(\alpha)}^{t} e^{a(t-\tau)}\left(f_{2}(\tau)+u^{\alpha}(\tau)\right) d \tau-\left[e^{a\left(t-t_{1}\right)} y_{2}+\int_{t_{1}}^{t} e^{a(t-\tau)}\left(f_{2}(\tau)+\bar{u}(\tau)\right) d \tau\right]}{\alpha} \\
& =\lim _{\alpha \rightarrow 0} \frac{\left(e^{a\left(t-h_{t_{1}}(\alpha)\right)}-e^{a\left(t-t_{1}\right)}\right) \tilde{y}(\alpha)}{h_{t_{1}}(\alpha)-t_{1}} \frac{h_{t_{1}}(\alpha)-t_{1}}{\alpha}+\lim _{\alpha \rightarrow 0} \frac{e^{a\left(t-t_{1}\right)}\left(\tilde{y}(\alpha)-y_{2}\right)}{\alpha} \\
& +\lim _{\alpha \rightarrow 0} \frac{\int_{h_{t_{1}}(\alpha)}^{t_{1}} e^{a(t-\tau)}\left(f_{2}(\tau)+\bar{u}(\tau)\right) d \tau}{h_{t_{1}}(\alpha)-t_{1}} \frac{h_{t_{1}}(\alpha)-t_{1}}{\alpha}+\lim _{\alpha \rightarrow 0} \int_{h_{t_{1}}(\alpha)}^{t} e^{a(t-\tau)} u(\tau) d \tau \\
& =\lim _{\alpha \rightarrow 0} \frac{\left(e^{a\left(t-h_{t_{1}}(\alpha)\right)}-e^{a\left(t-t_{1}\right)}\right) \tilde{y}(\alpha)}{h_{t_{1}}(\alpha)-t_{1}} \frac{h_{t_{1}}(\alpha)-t_{1}}{\alpha}+\lim _{\alpha \rightarrow 0} \frac{e^{a\left(t-t_{1}\right)}\left(x_{\alpha}\left(h_{t_{1}}(\alpha) ; u^{\alpha}\right)-\bar{x}\left(t_{1} ; \bar{u}\right)\right)}{\alpha} \\
& +\lim _{\alpha \rightarrow 0} \frac{\int_{h_{t_{1}}(\alpha)}^{t_{1}} e^{a(t-\tau)}\left(f_{2}(\tau)+\bar{u}(\tau)\right) d \tau}{h_{t_{1}}(\alpha)-t_{1}} \frac{h_{t_{1}}(\alpha)-t_{1}}{\alpha}+\lim _{\alpha \rightarrow 0} \int_{h_{t_{1}}(\alpha)}^{t} e^{a(t-\tau)} u(\tau) d \tau \\
& =\int_{t_{1}}^{t} e^{a\left(t_{1}-\tau\right)} u(\tau) d \tau+\dot{h}_{t_{1}}(0)\left(f_{1}\left(t_{1}\right)-f_{2}\left(t_{1}\right)\right)+\varphi\left(t_{1} ; u^{\alpha}\right) \\
& =\int_{t_{1}}^{t} e^{a\left(t_{1}-\tau\right)} u(\tau) d \tau+\varphi\left(t_{1}+; u^{\alpha}\right), \quad \forall t \in\left(t_{1}, t_{2}\right),
\end{aligned}
$$

we know from $(17)$ that $\varphi\left(\cdot ; u^{\alpha}\right)$ satisfies the following equation:

$$
\left\{\begin{array}{l}
\dot{\varphi}\left(t ; u^{\alpha}\right)=a \varphi\left(t ; u^{\alpha}\right)+u(t), \quad t \in\left(t_{1}, t_{2}\right), \\
\varphi\left(t_{1}+; u^{\alpha}\right)=\dot{h}_{t_{1}}(0)\left(f_{1}\left(t_{1}\right)-f_{2}\left(t_{1}\right)\right)+\varphi\left(t_{1} ; u^{\alpha}\right) .
\end{array}\right.
$$

Analogous to $\varphi\left(t_{1}+; u^{\alpha}\right)$, from equations (3), (9), and Lemma 3.1, we have the following results about $\varphi\left(t_{2}+; u^{\alpha}\right)$ : when $h_{t_{2}}(\alpha)>t_{2}$,

$$
\begin{aligned}
\varphi( & \left.t_{2}+; u^{\alpha}\right) \\
= & \lim _{\alpha \rightarrow 0} \frac{x_{\alpha}\left(h_{t_{2}}(\alpha) ; u^{\alpha}\right)-\bar{x}\left(h_{t_{2}}(\alpha) ; \bar{u}\right)}{\alpha} \\
= & \lim _{\alpha \rightarrow 0} \frac{x_{\alpha}\left(h_{t_{2}}(\alpha) ; u^{\alpha}\right)-\bar{x}\left(t_{2} ; \bar{u}\right)}{\alpha}+\lim _{\alpha \rightarrow 0} \frac{\bar{x}\left(t_{2} ; \bar{u}\right)-\bar{x}\left(h_{t_{2}}(\alpha) ; \bar{u}\right)}{\alpha} \\
= & \lim _{\alpha \rightarrow 0} \frac{x_{\alpha}\left(h_{t_{2}}(\alpha) ; u^{\alpha}\right)-x_{\alpha}\left(t_{2} ; u^{\alpha}\right)}{\alpha}+\lim _{\alpha \rightarrow 0} \frac{x_{\alpha}\left(t_{2} ; u^{\alpha}\right)-\bar{x}\left(t_{2} ; \bar{u}\right)}{\alpha} \\
& +\lim _{\alpha \rightarrow 0} \frac{\bar{x}\left(t_{2} ; \bar{u}\right)-\bar{x}\left(h_{t_{2}}(\alpha) ; \bar{u}\right)}{\alpha} \\
= & \lim _{\alpha \rightarrow 0} \frac{x_{\alpha}\left(h_{t_{2}}(\alpha) ; u^{\alpha}\right)-x_{\alpha}\left(t_{2} ; u^{\alpha}\right)}{h_{t_{2}}(\alpha)-t_{2}} \frac{h_{t_{2}}(\alpha)-t_{2}}{\alpha}+\lim _{\alpha \rightarrow 0} \frac{x_{\alpha}\left(t_{2} ; u^{\alpha}\right)-\bar{x}\left(t_{2} ; \bar{u}\right)}{\alpha} \\
& +\lim _{\alpha \rightarrow 0} \frac{\bar{x}\left(t_{2} ; \bar{u}\right)-\bar{x}\left(h_{t_{2}}(\alpha) ; \bar{u}\right)}{h_{t_{2}}(\alpha)-t_{2}} \frac{h_{t_{2}}(\alpha)-t_{2}}{\alpha} \\
= & h_{t_{2}}(0)\left(f_{2}\left(t_{2}\right)-f_{1}\left(t_{2}\right)\right)+\varphi\left(t_{2} ; u^{\alpha}\right)
\end{aligned}
$$


and when $h_{t_{2}}(\alpha)<t_{2}$,

$$
\begin{aligned}
\varphi\left(t_{2}+; u^{\alpha}\right)= & \lim _{\alpha \rightarrow 0} \frac{x_{\alpha}\left(t_{2} ; u^{\alpha}\right)-\bar{x}\left(t_{2} ; \bar{u}\right)}{\alpha} \\
= & \lim _{\alpha \rightarrow 0} \frac{x_{\alpha}\left(t_{2} ; u^{\alpha}\right)-x_{\alpha}\left(h_{t_{2}}(\alpha) ; u^{\alpha}\right)}{\alpha}+\lim _{\alpha \rightarrow 0} \frac{x_{\alpha}\left(h_{t_{2}}(\alpha) ; u^{\alpha}\right)-\bar{x}\left(h_{t_{2}}(\alpha) ; \bar{u}\right)}{\alpha} \\
& +\lim _{\alpha \rightarrow 0} \frac{\bar{x}\left(h_{t_{2}}(\alpha) ; \bar{u}\right)-\bar{x}\left(t_{2} ; \bar{u}\right)}{\alpha} \\
= & \lim _{\alpha \rightarrow 0} \frac{x_{\alpha}\left(t_{2} ; u^{\alpha}\right)-x_{\alpha}\left(h_{t_{2}}(\alpha) ; u^{\alpha}\right)}{h_{t_{2}}(\alpha)-t_{2}} \frac{h_{t_{2}}(\alpha)-t_{2}}{\alpha} \\
& +\lim _{\alpha \rightarrow 0} \frac{\bar{x}\left(h_{t_{2}}(\alpha) ; \bar{u}\right)-\bar{x}\left(t_{2} ; \bar{u}\right)}{h_{t_{2}}(\alpha)-t_{2}} \frac{h_{t_{2}}(\alpha)-t_{2}}{\alpha} \\
& +\varphi\left(t_{2} ; u^{\alpha}\right) \\
= & \dot{h}_{t_{2}}(0)\left(f_{2}\left(t_{2}\right)-f_{1}\left(t_{2}\right)\right)+\varphi\left(t_{2} ; u^{\alpha}\right) .
\end{aligned}
$$

The above equations yield that

$$
\varphi\left(t_{2}+; u^{\alpha}\right)=\dot{h}_{t_{2}}(0)\left(f_{2}\left(t_{2}\right)-f_{1}\left(t_{2}\right)\right)+\varphi\left(t_{2} ; u^{\alpha}\right) .
$$

Repeat the above process, we consider the time interval $\left(t_{m}, t_{m}+1\right)(m=1,2, \ldots, k-1)$ or $\left(t_{k}, T\right)$. Firstly, we have the following equation:

$$
\begin{aligned}
& \varphi\left(t ; u^{\alpha}\right) \\
& =\lim _{\alpha \rightarrow 0} \frac{x\left(t ; u^{\alpha}\right)-\bar{x}(t ; \bar{u})}{\alpha} \\
& =\lim _{\alpha \rightarrow 0} \frac{e^{a\left(t-h_{t_{m}}(\alpha)\right)} \tilde{y}(\alpha)+\int_{h_{t_{m}}(\alpha)}^{t} e^{a(t-\tau)}\left(f_{i}(\tau)+u^{\alpha}(\tau)\right) d \tau-\left[e^{a\left(t-t_{m}\right)} y_{i}+\int_{t_{m}}^{t} e^{a(t-\tau)}\left(f_{i}(\tau)+\bar{u}(\tau)\right) d \tau\right]}{\alpha} \\
& =\lim _{\alpha \rightarrow 0} \frac{\left(e^{a\left(t-h_{t_{m}}(\alpha)\right)}-e^{a\left(t-t_{m}\right)}\right) \tilde{y}(\alpha)}{h_{t_{m}}(\alpha)-t_{m}} \frac{h_{t_{m}}(\alpha)-t_{m}}{\alpha}+\lim _{\alpha \rightarrow 0} \frac{e^{a\left(t-t_{m}\right)}\left(\tilde{y}(\alpha)-y_{i}\right)}{\alpha} \\
& +\lim _{\alpha \rightarrow 0} \frac{\int_{h_{t_{m}}(\alpha)}^{t_{m}} e^{a(t-\tau)}\left(f_{i}(\tau)+\bar{u}(\tau)\right) d \tau}{h_{t_{m}}(\alpha)-t_{m}} \frac{h_{t_{m}}(\alpha)-t_{m}}{\alpha}+\lim _{\alpha \rightarrow 0} \int_{h_{t_{m}}(\alpha)}^{t} e^{a(t-\tau)} u(\tau) d \tau \\
& =\lim _{\alpha \rightarrow 0} \frac{\left(e^{a\left(t-h_{t_{m}}(\alpha)\right)}-e^{a\left(t-t_{m}\right)}\right) \tilde{y}(\alpha)}{h_{t_{m}}(\alpha)-t_{m}} \frac{h_{t_{m}}(\alpha)-t_{m}}{\alpha}+\lim _{\alpha \rightarrow 0} \frac{e^{a\left(t-t_{m}\right)}\left(x_{\alpha}\left(h_{t_{m}}(\alpha) ; u^{\alpha}\right)-\bar{x}\left(t_{m} ; \bar{u}\right)\right)}{\alpha} \\
& +\lim _{\alpha \rightarrow 0} \frac{\int_{h_{t_{m}}(\alpha)}^{t_{m}} e^{a(t-\tau)}\left(f_{i}(\tau)+\bar{u}(\tau)\right) d \tau}{h_{t_{m}}(\alpha)-t_{m}} \frac{h_{t_{m}}(\alpha)-t_{m}}{\alpha}+\lim _{\alpha \rightarrow 0} \int_{h_{t_{m}}(\alpha)}^{t} e^{a(t-\tau)} u(\tau) d \tau \\
& =\int_{t_{m}}^{t} e^{a\left(t_{m}-\tau\right)} u(\tau) d \tau+\dot{h}_{t_{m}}(0)\left(f_{j}\left(t_{m}\right)-f_{i}\left(t_{1}\right)\right)+\varphi\left(t_{m} ; u^{\alpha}\right) \\
& =\int_{t_{m}}^{t} e^{a\left(t_{m}-\tau\right)} u(\tau) d \tau+\varphi\left(t_{m}+; u^{\alpha}\right), \quad \forall t \in\left(t_{m}, t_{m}+1\right) \cup\left(t_{k}, T\right),
\end{aligned}
$$

where $i=2, j=1$ if $m$ is even and $i=1, j=2$ if $m$ is odd. The above equation implies that, for any $t \in\left(t_{m}, t_{m}+1\right) \cup\left(t_{k}, T\right)(m=1,2, \ldots, k-1), \varphi\left(t ; u^{\alpha}\right)$ satisfies the following differential 
equation:

$$
\dot{\varphi}\left(t ; u^{\alpha}\right)=a \varphi\left(t ; u^{\alpha}\right)+u(t), \quad t \in\left(t_{m}, t_{m}+1\right) \cup\left(t_{k}, T\right) .
$$

On the other hand, analogous to $\varphi\left(t_{1}+; u^{\alpha}\right)$ and $\varphi\left(t_{2}+; u^{\alpha}\right)$, we obtain that

$$
\varphi\left(t_{m+1}+; u^{\alpha}\right)=\dot{h}_{t_{m+1}}(0)\left(f_{i}\left(t_{m+1}\right)-f_{j}\left(t_{m+1}\right)\right)+\varphi\left(t_{m+1} ; u^{\alpha}\right),
$$

where $i=2, j=1$ if $m$ is even and $i=1, j=2$ if $m$ is odd.

In conclusion, we can deduce from (18), (19), and (20) that $\varphi\left(\cdot ; u^{\alpha}\right)$ satisfies the following impulsive differential equation:

$$
\begin{cases}\dot{\varphi}\left(t ; u^{\alpha}\right)=a \varphi\left(t ; u^{\alpha}\right)+u(t), & t \in\left(t_{0}, T\right], x(t) \neq y_{p}(p \in \mathscr{P}), \\ \varphi\left(t+; u^{\alpha}\right)=\varphi\left(t ; u^{\alpha}\right)+\dot{h}_{t}(0)\left[f_{j}(t)-f_{i}(t)\right], & x(t)=y_{i}(i, j \in \mathscr{P}, i \neq j), \\ \varphi\left(0 ; u^{\alpha}\right)=0 . & \end{cases}
$$

The proof of Theorem 3.1 is complete.

\section{Conclusions}

We considered a class of switched systems with internally forced switching. This system may be helpful for modeling the intelligent system, such as the self-driving system, switched Hopfield neural network, memristive neural network, etc. Naturally, the optimal control problem of such systems is a hot topic in applied mathematics. However, the existing literature of this problem mainly focuses on a special class controlled system (the piece affine system). There are few reports about more general internally forced switching problem (the optimal control problem given by IFS), especially in theory. The principal reason for this is that the internally forced switching law can destroy the continuous dependence of the solution with respect to the control (see IFS (3) for details), which leads to the fact that the classical optimal control theory is not suitable for more general internally forced switched systems. Hence, in the paper, we investigate the continuous dependence and the differentiability of the solution with respect to the control. These results lay the foundation for studying a more general internally forced switching problem. Our future research directions are (i) extending the presented results to the more general IFS and (ii) studying the optimal control problem given by IFS (2) by using the presented results.

Acknowledgements

The authors thank the anonymous referees for their expert advice and suggestions, which helped to improve this paper.

Funding

This research was funded by the Foundation of Postgraduate of Guizhou Province grant number 2019032.

Abbreviations

IFS, Switched system with internally forced switching; EFS, Switched system with externally forced switching.

Availability of data and materials

Not applicable.

\section{Declarations}




\section{Authors' contributions}

Formal analysis, HL and XC; Investigation, HL and XC; Writing —original draft preparation, HL and XC. The authors contributed equally to this work. All authors have read and agreed to the published version of the manuscript.

\section{Author details}

'Department of Mathematics, Guizhou University, Guiyang, China. ${ }^{2}$ Department of Mathematics and Computer Science, Liupanshui Normal University, Liupanshui, China.

\section{Publisher's Note}

Springer Nature remains neutral with regard to jurisdictional claims in published maps and institutional affiliations.

\section{Received: 9 June 2021 Accepted: 10 October 2021 Published online: 12 November 2021}

\section{References}

1. Allerhand, L.I., Shaked, U.: Stability of stochastic nonlinear systems with state-dependent switching. IEEE Trans. Autom. Control 58, 994-1001 (2013)

2. Bemporad, A., Giua, A., Seatzu, C.: A master-slave algorithm for the optimal control of continuous-time switched affine systems. In: Proceedings of the 41st IEEE Conference on Decision and Control, Las Vegas (2002)

3. Brockett, R.W.: Hybrid models for motion control systems. In: Essays on Control: Perspectives in the Theory and Its Applications. Bikhäuser, Boston (1993)

4. Cai, C., Teel, A.: Robust input-to-state stability for hybrid systems. SIAM J. Control Optim. 51, 1651-1678 (2013)

5. Cui, S., Liu, C., Liu, X.: On the general problem of solution and stability for variable-time switched systems. In: 2017 International Workshop on Complex Systems and Networks, Doha (2017)

6. Cui, S., Liu, C., Liu, X.: On the general problem of solution and stability for variable-time switched systems. In: 2017 International Workshop on Complex Systems and Networks, Doha (2017)

7. Deane, J.H.B., Hamill, D.C.: Instability, subharmonics, and chaos in power electronic systems. IEEE Trans. Power Electron. 5, 260-268 (1990)

8. Ding, K., Zhu, Q.: Extended dissipative anti-disturbance control for delayed switched singular semi-Markovian jump systems with multi-disturbance via disturbance observer. Automatica 128, 109556 (2021)

9. Du, H., Wen, G., Wu, D., Cheng, Y., Lü, J., Bemporad, A., Ljung, L.: Distributed fixed-time consensus for nonlinear heterogeneous multi-agent systems. Automatica 113, 108797 (2020)

10. Egerstedt, M., Wardi, Y., Delmotte, F.: Optimal control of switching times in switched dynamical systems. In: Proceedings of the 42nd IEEE Conference on Decision and Control, Maui (2003)

11. Engell, S., Kowalewski, S., Schulz, C., Stursberg, O.: Continuous-discrete interactions in chemical processing plants. Proc. IEEE 88, 1050-1068 (2000)

12. Gokbayrak, K., Cassandras, C.G.: A hierarchical decomposition method for optimal control of hybrid systems. In: Proceedings of the 39th IEEE Conference on Decision and Control, Sydney (2000)

13. Hiskens, L.: Stability of hybrid system limit cycles: application to the compass gait biped robot. In: Decision and Control, Proceeding of the 40th IEEE Conference on Decision and Control, Orlando (2001)

14. Hopfield, J.J.: Neurons with graded response have collective computational properties like those of two-state neurons. Proc. Natl. Acad. Sci. USA 81, 3088-3092 (1984)

15. Imura, J., Schaft, A.: Characterization of well-posedness of piecewise-linear systems. IEEE Trans. Autom. Control 45 $1600-1619(2000)$

16. Jeon, D., Tomizuka, M.: Learning hybrid force and position control of robot manipulators. IEEE Trans. Robot. Autom. 9 , 423-431 (1993)

17. Johnson, E., Murphey, T:: Second-order switching time optimization for nonlinear time-varying dynamic systems. IEEE Trans. Autom. Control 56, 1953-1957 (2011)

18. Kerrigan, E., Mayne, D.: Optimal control of constrained, piecewise affine systems with bounded disturbances. In: Proceedings of the 41 st IEEE Conference on Decision and Control, Las Vegas (2002)

19. King, C.K., Griggs, W.M., Shorten, R.N.: A Result on the Existence of Quadratic Lyapunov Functions for State-Dependent Switched Systems with Uncertainty. 49th IEEE Conference on Decision and Control (CDC). Atlanta (2010)

20. Kong, F., Zhu, Q., Huang, T.: New fixed-time stability lemmas and applications to the discontinuous fuzzy inertial neural networks. IEEE Trans. Fuzzy Syst. (2020). https://doi.org/10.1109/TFUZZ.2020.3026030

21. Kong, F., Zhu, Q., Huang, T.: Fixed-time stability for discontinuous uncertain inertial neural networks with time-varying delays. IEEE Trans. Syst. Man Cybern. Syst., 1-11 (2021). https://doi.org/10.1109/TSMC.2021.3096261

22. Lennartson, B., Tittus, M., Egardt, B.: Hybrid systems in process control. IEEE Control Syst. Mag. 16, 45-56 (2002)

23. Li, H., Peng, Y., Wu, K.: Properties of solution for the nonlinear on-off switched differential equation with switching at variable times. Nonlinear Dyn. 103, 2287-2298 (2021)

24. Lin, H., Antsaklis, P.: Stability and stabilizability of switched linear systems: a survey of recent results. IEEE Trans. Autom. Control 54, 308-322 (2000)

25. Liu, C., Yang, Z., Sun, D., Liu, X.: Stability of variable-time switched systems. Arab. J. Sci. Eng. 42, 2971-2980 (2017)

26. Liu, C., Yang, Z., Sun, D., Liu, X., Liu, W.: Stability of switched neural networks with time-varying delays. Neural Comput. Appl. 30, 2229-2244 (2018)

27. Liu, S., Liberzon, D.: Global Stability and Asymptotic Gain Imply Input-to-State Stability for State-Dependent Switched Systems. 2018 IEEE Conference on Decision and Control (CDC). Miami (2018)

28. Mignone, D., Ferrari-Trecate, G., Morari, M.: Stability and stabilization of piecewise affine and hybrid systems: an LMI approach. In: Proceedings of the 39th IEEE Conference on Decision and Control, Sydney (2000)

29. Piccoli, B.: Necessary conditions for hybrid optimization. In: Proceedings of the 38th IEEE Conference on Decision and Control, Phoenix (1999)

30. Riedinger, P., Kratz, F., Lung, C., Zanne, C.: Linear quadratic optimization for hybrid systems. In: Proceedings of the 38th IEEE Conference on Decision and Control, Phoenix (1999) 
31. Roll, J., Bemporad, A., Ljung, L.: Identification of piecewise affine systems via mixed-integer programming. Automatica 40, 37-50 (2004)

32. Sanders, S.R., Verghese, G.C.: Lyapunov-based control for switched power converters. IEEE Trans. Power Electron. 7, 17-24 (1992)

33. Shorten, R., Wirth, F., Mason, O., Kai, W., King, C.: Stability criteria for switched and hybrid systems. SIAM Rev. 49, 545-592 (2007)

34. Sussmann, H.J.: Set-valued differentials and the hybrid maximum principle. In: Proceedings of the 39th IEEE Conference on Decision and Control, Sydney (2000)

35. Wang, B., Zhu, Q.: Stability analysis of discrete-time semi-Markov jump linear systems. IEEE Trans. Autom. Control 65 5415-5421 (2020)

36. Wyczalek, F.A.: Hybrid electric vehicles: year 2000 status. IEEE Aerosp. Electron. Syst. Mag. 16, 15-25 (2001)

37. Xu, X., Antsaklis, P.: A dynamic programming approach for optimal control of switched systems. In: Proceedings of the 39th IEEE Conference on Decision and Control, Sydney (2000)

38. $\mathrm{Xu}, \mathrm{X}$. Antsaklis, P.: Optimal control of switched systems: new results and open problem. In: Proceedings of the 2000 American Control Conference (ACC), Chicago (2000)

39. $\mathrm{Xu}, \mathrm{X}$., Antsaklis, P:: Optimal control of switched systems based on parameterization of the switching instants. IEEE Trans. Autom. Control 1, 2-16 (2004)

40. Zhang, G., Shen, Y: Novel conditions on exponential stability of a class of delayed neural networks with state-dependent switching. Neural Netw. 71, 55-61 (2015)

41. Zhang, X., Li, C., Huang, T.: Hybrid impulsive and switching Hopfield neural networks with state-dependent impulses. Neural Netw. 93, 176-184 (2017)

42. Zhu, Q: Stabilization of stochastic nonlinear delay systems with exogenous disturbances and the event-triggered feedback control. IEEE Trans. Autom. Control 64, 3764-3771 (2019)

\section{Submit your manuscript to a SpringerOpen ${ }^{\circ}$ journal and benefit from:}

- Convenient online submission

- Rigorous peer review

- Open access: articles freely available online

- High visibility within the field

- Retaining the copyright to your article

Submit your next manuscript at $\gg$ springeropen.com 